\title{
1 Hollow Silica Microspheres for Buoyancy-Assisted Separation of Infectious Pathogens from Stool
}

\author{
3 Shannon E. Weigum ${ }^{1,2,{ }^{*}}$, Lichen Xiang $^{1}$, Erica Osta ${ }^{2}$, Linying Li ${ }^{3,4}$, and Gabriel P. López ${ }^{3,4,5}$ \\ 1 Materials Science, Engineering, and Commercialization Program, Texas State University, \\ San Marcos, TX 78666, USA. \\ 27708, USA. \\ 5 Center for Biomedical Engineering, Department of Chemical and Biomedical Engineering,
}

\section{ABSTRACT}

Separation of cells and microorganisms from complex biological mixtures is a critical first step in many analytical applications ranging from clinical diagnostics to environmental monitoring for food and waterborne contaminants. Yet, existing techniques for cell separation are plagued by high reagent and/or instrumentation costs that limit their use in many remote or resource-poor settings, such as field clinics or developing countries. We developed an innovative approach to isolate infectious pathogens from biological fluids using buoyant hollow silica microspheres that function as "molecular buoys" for affinity-based target capture and separation by floatation. In this process, antibody functionalized glass microspheres are mixed with a complex biological sample, such as stool. When mixing is stopped, the target-bound, low-density microspheres float to the air/liquid surface, which simultaneously isolates and concentrates the target analytes from the sample matrix. The microspheres are highly tunable in terms of size, density, and surface functionality for targeting diverse analytes with separation times of $\leq 2$ minutes in viscous solutions. We have applied the molecular buoy technique for isolation of a protozoan parasite that causes diarrheal illness, Cryptosporidium, directly from stool with separation efficiencies over $90 \%$ and low non-specific binding. This low-cost method for phenotypic cell/pathogen separation from complex mixtures is expected to have widespread use in clinical diagnostics as well as basic research.

Keywords: Bioseparation, sample preparation, analyte concentration and isolation, buoyancy cell separation, glass microbubbles

*Corresponding Author: Shannon Weigum, email: sweigum@txstate.edu 


\section{INTRODUCTION}

Separation of disease-related cells and microorganisms from complex biological mixtures

40 is a critical first step in many analytical applications ranging from clinical diagnostics to

41 environmental monitoring for food and waterborne pathogens [1]. Existing separation methods

42 can be loosely grouped into two categories; those that rely upon physical differences among cells

43 (i.e. size, shape, density, or electrophoretic mobility); and those that rely upon differences in

44 chemical composition (i.e. protein or other cell surface markers) [1,2]. The most common

45 physical separation methods include size-dependent micro/ultrafiltration and density-based

46 centrifugation using Percoll $^{\circledR}$ or Ficoll-Paque ${ }^{\circledR}$ density gradients $[1,3]$. These methods typically

47 have the advantage of being label-free and non-destructive which allows for subsequent cell

48 culture or growth; however, they depend upon a large physical difference between target and

49 non-target materials, limiting the ability to discriminate between closely-related populations [2].

50 Affinity-based techniques, such as magnetic-activated cell separation (MACS) and fluorescence-

51 activated cell separation (FACS) overcome these limitations through the use of target-specific

52 antibodies to impart higher selectivity for the particular cell type or pathogen of interest $[2,4,5]$;

53 however, high reagent and/or instrumentation costs continue to limit their use in many remote or

54 resource-poor settings. Even microfluidic and lab-on-a-chip platforms for cell separation remain

55 limited in their widespread clinical adoption due to complexity and cost.

56 Recently, a few studies have emerged describing a buoyancy-activated cell sorting

57 (BACS) technique that uses low-density hollow glass microbubbles for separation of CD4+ T-

58 cells and circulating tumor cells from whole blood, and even cancer stem cells from

59 heterogeneous cell populations [6-8]. Advantages of BACS include (i) low material cost of the

60 glass microbubbles, which are an industrial additive used in plastics and coatings available in 
61 kilogram quantities, and (ii) the simplicity of the floatation-based separation, which eliminates

62 the need for any external instrumentation like a centrifuge or magnet. In addition, surface

63 functionalization of glass microbubbles can take advantage of the well-established silica-based

64 chemistries for direct conjugation of proteins, antibodies, or other affinity ligand [9].

In the current study, we describe for the first time a BACS process for the isolation of an

66 infectious disease pathogen from perhaps the most complicated biological sample type, stool. A

67 schematic of this "molecular buoy" method for isolation of Cryptosporidium, a protozoan

68 parasite that causes diarrheal illness, is illustrated in Fig 1. Here, a complex biological sample is

69 incubated with functionalized hollow microspheres and mixed in order to facilitate target capture

70 of the analytes. When mixing is stopped, the target-bound low-density microspheres migrate to

71 the air/liquid interface where they float at the surface, which simultaneously isolates and

72 concentrates the target analytes from the sample matrix.

73 We chose Cryptosporidium as our initial target pathogen since passive fecal floatation is

74 a technique that has long been used for the recovery of parasitic eggs and oocysts in stool prior to

75 microscopic examination and diagnosis in humans and pets [10-12]. The conventional stool

76 floatation method relies on the differences in the specific gravity of the eggs/oocysts, fecal

77 debris, and the floatation solution [11]. Some common floatation solutions include modified

78 Sheather's solution, zinc sulfate and magnesium sulfate with a specific gravity in the range of

79 1.18-1.27 (specific gravity of water is 1.0) [11]. Parasitic eggs/oocysts that have a lower specific

80 gravity (between 1.05-1.15) will, over time or under centrifugal force, float to the surface while

81 heavier debris will sink to the bottom [11]. Drawbacks of this technique include low recovery

82 rates, the remaining need for centrifugation, and the constraint to organisms that have an

83 inherently low specific gravity, such as eggs and encapsulated cysts, which can have gas or air 
84 pockets that lower their overall density. Using the molecular buoy approach, we aim to overcome

85 these restrictions and generate a high affinity, density-based separation technique that uses low-

86 cost materials, eliminates any external instrumentation or centrifugation, and can be universally

87 applied to isolate a variety of clinical pathogens and biomarkers.

\section{MATERIALS AND METHODS}

\subsection{Materials and Reagents}

We obtained the $\mathrm{H} 20$ and $\mathrm{H} 50$ hollow silica microspheres, with an epoxy silane surface

91 treatment and a density of $0.2 \mathrm{~g} / \mathrm{cm}^{3}$ and $0.5 \mathrm{~g} / \mathrm{cm}^{3}$, respectively from $3 \mathrm{M}(\mathrm{H} 20 / \mathrm{H} 50$ glass

92 bubbles; 3M, St. Paul, MN). Buffers, glycerol, protein G, and L-cysteine were all purchased

93 from Thermo Fisher Scientific (Waltham, MA). Phosphate buffered saline (PBS) was prepared in

94 deionized water using BupH ${ }^{\mathrm{TM}}$ Modified Dulbecco’s PBS (\#28374) buffer packs with a final

95 concentration of $8 \mathrm{mM}$ sodium phosphate, $2 \mathrm{mM}$ potassium phosphate, $0.14 \mathrm{M}$ sodium chloride

96 and $10 \mathrm{mM}$ potassium chloride at a $\mathrm{pH}$ of 7.4. C. parvum oosysts (Iowa isolate, \#P102C) and

97 negative control stool were purchased from Waterborne Inc. (New Orleans, LA). Antibodies

98 included an unconjugated anti-C. parvum monoclonal mouse IgG3 (clone BEL 0126, \#64526,

99 Novus Biologicals, Littleton, CO), an anti-C. parvum directly conjugated to Alexa-Fluor ${ }^{\circledR} 488$

100 (clone BEL 0126, \#2402-3007AF488, Bio-Rad AbD Serotec, Raleigh, NC) and a goat-anti-

101 mouse (GAM) IgG conjugated to Alexa-Fluor ${ }^{\circledR} 488$ (\#A11001, Invitrogen, Carlsbad, CA).

$102 \quad 2.2 \quad$ Microsphere Size and Floatation Kinetics

The H20/H50 glass microspheres were suspended in PBS at a final concentration of 1.0

$104 \mathrm{mg} / \mathrm{ml}$. A wet-mount slide using $\sim 10 \mu 1$ of the microsphere solution was prepared for imaging on 105 an EVOS FL Auto Cell Imaging System (Thermo Scientific, Waltham, MA) using an Olympus 
UPlanApo 10X objective (0.4 NA). The diameter of at least 5,000 microspheres was measured

107 directly from transmitted light microscopy images using ImageJ open-source software [13].

108 ImageJ particle analysis criteria applied to binary, thresholded images included a minimum

109 circularity of 0.85 in order to eliminate non-solitary or broken microspheres from the size

110 analysis. Histograms with a bin size $4 \mu \mathrm{m}$ were prepared in SigmaPlot 12.5 (SyStat Software,

111 Inc., San Jose, CA), along with all other graphs.

Additional size fractionation of the $\mathrm{H} 20$ microspheres was achieved using a stacked set of

113 wire mesh test sieves (VWR, Radnor, PA) with standard mesh sizes of 140, 200, 270, and 500

114 that correspond to a nominal opening of $105 \mu \mathrm{m}, 74 \mu \mathrm{m}, 53 \mu \mathrm{m}$, and $25 \mu \mathrm{m}$, respectively. In this

115 process, $1 \mathrm{~g}$ of dry $\mathrm{H} 20$ microspheres were placed in the upper sieve and shaken overnight $(>12$

$116 \mathrm{hrs}$ ) on a rotary shaker at $350 \mathrm{rpm}$. Each fractionated population was decanted from the

117 respective sieve and stored at room temperature in clean glass vials.

118 The floatation and buoyancy properties of the silica microspheres were characterized

119 using a UV/Vis spectrophotometer (Cary 60 UV/Vis; Agilent Technologies, Santa Clara, CA) to

120 monitor the change in optical density (OD) at $450 \mathrm{~nm}$ over $10 \mathrm{~min}$ as the particles floated to the

121 surface of a $2 \mathrm{ml}$ quartz cuvette. Separation time was calculated from the kinetic data as the time

122 point at which $90 \%$ of the total OD decline occurred $\left(0.9 \times\left(\mathrm{OD}_{\text {initial }}-\mathrm{OD}_{\text {final }}\right)\right)$. The various

123 glycerol concentrations, from $0 \%-50 \%$ in PBS, were used in this characterization to simulate

124 the wide range of fluid densities and viscosities expected in biological fluids.

\section{$125 \quad 2.3 \quad$ Surface Functionalization}

The epoxy silane reactive groups were used to directly conjugate protein $\mathrm{G}$ to the

127 microsphere surface via a $\mathrm{pH}$-dependent ring-opening reaction [9]. Here, protein $\mathrm{G}(0.01 \mathrm{mg} / \mathrm{ml}$

$128-1.0 \mathrm{mg} / \mathrm{ml})$ was incubated with $1 \mathrm{mg}$ of the $25-53 \mu \mathrm{m}$ fractionated microspheres in $100 \mu \mathrm{l}(1.4$ 
$129 \times 10^{6}$ microspheres $\left./ \mathrm{ml}\right)$ of PBS buffer $(\mathrm{pH} 7.4)$ or $0.1 \mathrm{M}$ sodium carbonate/bicarbonate buffer at 130 various $\mathrm{pH}$ (range $9.1-10.5$ ) [14] for $24 \mathrm{hrs}$ at $4^{\circ} \mathrm{C}$ with continuous end-over-end mixing. L131 cysteine was added to a final concentration of $50 \mathrm{mM}$ and incubated for another $24 \mathrm{hrs}$ in order 132 to block any unreacted epoxy groups. Next, we washed the microspheres three times in PBS by 133 tilting the microfuge tube $\sim 45^{\circ}$ allowing the particles to collect at the liquid surface, then 134 removing the lower solute fraction and resuspending remaining particles in $100-200 \mu 1$ buffer. 135 Functionalized particles were typically used immediately or stored at $4^{\circ} \mathrm{C}$ for up to one week.

136 The presence of protein $\mathrm{G}$ on the microsphere surface was confirmed indirectly by 137 fluorescence microscopy and quantitative image analysis of protein $\mathrm{G}$ bound to a secondary 138 GAM/Alexa-Fluor ${ }^{\circledR} 488$ antibody. GAM binding was carried out at a final concentration of 0.1 $139 \mathrm{mg} / \mathrm{ml}$ in PBS, mixed with $0.1 \mathrm{mg}$ protein $\mathrm{G}$-functionalized microspheres for $2 \mathrm{hrs}$ at room 140 temperature. Epi-fluorescent images were collected from at least 10 fields-of-view and exported 141 to ImageJ for quantitative analysis. Analysis routines included automatic intensity thresholding, 142 conversion to a binary mask, holes filled, and watershed applied to obtain region-of-interest 143 (ROI) outlines for each particle with a minimum size of 500 pixels and circularity of 0.75 . The 144 intensities within the ROIs were measured on the original 8-bit grayscale image to obtain mean 145 fluorescence intensity (MFI) per microsphere. Approximately 2,000 particles were measured at 146 each protein $\mathrm{G}$ concentration run in triplicate. Data was graphed in SigmaPlot and fit to an 147 exponential rise-to-maximum curve with equation: $f=y_{0}+a\left(1-e^{-b x}\right)$. Microsphere 148 concentrations (beads/ml and beads $/ \mathrm{mg}$ ) were obtained from hemocytometer counts of $1 \mathrm{mg}$ of 149 beads from the $25-53 \mu \mathrm{m}$ fraction resuspended in $100 \mu 150 \%$ glycerol/PBS. 


\subsection{Capture of Cryptosporidium parvum oocysts}

Protein G-coated microspheres were prepared as described above using $0.1 \mathrm{mg} / \mathrm{ml}$ protein

$152 \mathrm{G}$ in $100 \mu \mathrm{l}$ sodium carbonate/bicarbonate buffer at $\mathrm{pH} \leq 9.4$. Blank control microspheres did not

153 contain protein $\mathrm{G}$ (i.e. buffer only). For capture studies, protein G-coated and control

154 microspheres were incubated with unconjugated anti-Cryptosporidium $\mathrm{IgG}(0.01 \mathrm{mg} / \mathrm{ml}$ in PBS)

155 for $2 \mathrm{hrs}$ at room temperature and washed three times by floatation. Microspheres were then 156 mixed with either buffer or stool samples spiked with $10^{2}-10^{5}$ oocysts in $100 \mu \mathrm{l}$ for up to $2 \mathrm{hrs}$

157 with continuous end-over-end mixing. Following a 1-2 min floatation-based separation step, the

158 lower fraction was transferred to a new microfuge tube where any unbound oocysts were counted

159 using a hemocytometer. At the lower oocyst concentrations, centrifugation and resuspension of

160 the recovered fraction (5,000 rpm for $5 \mathrm{~min}$, supernatant removed and pellet resuspended in 20

$161 \mu \mathrm{l})$ was needed for counting. The difference between the total number of oocysts input into each

162 sample and the number recovered in the un-bound fraction was used to calculate the percent

163 oocysts bound by the functionalized molecular buoys. Three independent replicates at each

164 oocyst concentration were run in buffer and two in stool. A one-tailed t-test was performed in

165 SigmaPlot to determine significant differences between positive/negative control samples and

166 between PBS/stool samples containing the same number of oocysts $(p<0.05)$. Negative control

167 stool was purchased from a commercial vendor, Waterborne Inc. (New Orleans, LA) and

168 consisted of roughly $10 \%$ insoluble solid material and $90 \%$ water. Since Cryptosporidium

169 infection causes diarrhea, characterized by loose or watery stool with a water content $>80 \%$ [15],

170 the stool sample used in this study closely mimicked this condition without further dilution or

171 solubilization. 
173 Fluor $^{\circledR} 488$ conjugate of the same monoclonal antibody clone at $0.1 \mathrm{mg} / \mathrm{ml}$ in PBS. In addition,

174 the Cryptosporidium oocysts were added immediately after the $2 \mathrm{hr}$ antibody incubation, without

175 washing excess antibody from the functionalized microspheres. Thus, capture and

176 immunolabeling of oocysts was allowed to occur simultaneously and enabled visualization of

177 both the microspheres and bound Cryptosporidium via fluorescence. Confocal imaging was

178 performed on an Olympus FV100 Scanning Confocal Microscope with a 60X PlanApoN oil-

179 immersion objective (1.4 NA) (Olympus Corporation, Tokyo, Japan). Alternatively, we pre-

180 labeled and washed the oocysts $\left(0.01 \mathrm{mg} / \mathrm{ml}\right.$ anti-Crypto/Alexa-Fluor ${ }^{\circledR} 488$ for 2 hrs followed by

181 centrifugation at 5,000 rpm, supernatant removed) then added them to protein G-functionalized

182 microspheres for capture of the antibody:oocyst immunocomplexes, followed by epi-

183 fluorescence imaging as previously described.

184 3. RESULTS AND DISCUSSION

\section{$185 \quad 3.1 \quad$ Characterization of size and buoyancy properties}

186 We measured the diameter of H20 and H50 microspheres from microscopy images and

187 generated histogram plots to examine the distribution of bead sizes present in each population.

188 We found a wide range of particle sizes in the $\mathrm{H} 20$ population, from less than $10 \mu \mathrm{m}$ in diameter

189 to more than $80 \mu \mathrm{m}$ in diameter, with a population mean of $27 \pm 13 \mu \mathrm{m}$ and a mode of $16 \mu \mathrm{m}$

190 (Fig 2A). The H50 microspheres were slightly smaller with a mean diameter of $17 \pm 7 \mu \mathrm{m}$ and a

191 mode of $12 \mu \mathrm{m}$ (Fig 2B).

192 Next, we monitored the rate of microsphere separation by time-lapse video and optical

193 spectroscopy (Fig 3). In PBS buffer, the H20 microspheres immediately began to migrate 
194 upward when mixing was stopped and, after roughly 2 min, nearly all of the microspheres could

195 be seen floating at the surface with cleared lower fluid fraction (Fig 3A; full video available in

196 Supplemental Material). Kinetic traces monitoring the change in optical density throughout this

197 floatation-based separation process in PBS buffer, and solutions containing increasing amounts

198 of glycerol, are shown in Fig 3B and 3C. The fastest decline in OD was detected in PBS buffer,

199 or 0\% glycerol, indicating the highest separation rate in this solution with low density, low

200 viscosity. As glycerol content increased up to 50\%, the rate of OD decline was progressively

201 delayed for both the H20 and H50 glass bubbles. Using the kinetic data, we calculated the

202 experimental separation time as the time point at which $90 \%$ of the total OD change had already

203 occurred. For the H20 glass microspheres, the separation time was 2.7 min in PBS buffer and 6.7

$204 \mathrm{~min}$ in the 50\% glycerol/PBS solution, while the slightly smaller H50 glass microspheres had a

205 separation time of $4.7 \mathrm{~min}$ in buffer and $8.2 \mathrm{~min}$ in $50 \%$ glycerol. A plot $\mathrm{H} 20$ separation time

206 versus fluid viscosity closely followed the non-linear relationship between glycerol content and

207 viscosity (Fig 3B, inset), suggesting that viscosity is a key parameter influencing separation time

208 and efficiency. A summary of all experimentally derived separation times is provided in

209 Supplemental Table 1, along with published values for fluid viscosity and density of the glycerol

210 solutions used $[16,17]$.

\section{$211 \quad 3.1 .1 \quad$ Theoretical considerations}

212 These results can be better understood by revisiting a few principles of physics which

213 describe the three forces acting on the particles (Fig 4). For a submerged object, the upward

214 buoyant force is equal to the weight of the displaced fluid (Archimedes' principle) and can be

215 calculated according to Equation 1 where $\rho_{f}$ is the density of the fluid, $V_{f}$ is the volume of the

216 displaced fluid, and $g$ is gravity $\left(9.8 \mathrm{~m} / \mathrm{s}^{2}\right)$. Submerged objects that rise in a fluid have a buoyant 
217 force that is greater than the sum of the two downward forces due to gravity and drag. The force

218 of gravity is simply the weight of the object (mg), and can be written according to Equation 2

219 where $\rho_{o}$ is the density of the object, $V_{o}$ is the volume of the object, and $g$ is gravity. The drag

220 force, also known as the frictional force or Stokes' drag, on the object is given by Equation 3

221 where $\eta$ is the fluid viscosity, $r$ is the radius of a spherical object, and $v$ is the velocity [18].

222 When the net force is positive $\left(F_{\text {buoyancy }}>F_{\text {grav }}+F_{\text {drag }}\right)$, the object will accelerate upwards until

223 the object reaches equilibrium where the net force is zero (the sum of forces $F_{n e t}=0$ ) and the

224 object is rising at a constant speed or it is floating on the surface of the fluid. Since the silica

225 microspheres are hollow, the buoyant force is largely size-dependent with larger particles having

226 less density and displacing a larger volume of fluid than smaller microspheres in a fluid with

227 similar density/viscosity. As such, the magnitude of the positive net force is greater, despite an

228 increase in both the gravity and drag force for larger particles than smaller particles. For

229 example, the buoyant force acting upon the $\mathrm{H} 20$ microspheres with a mass of $2.06 \mathrm{ng}$ is $1.01 \mathrm{x}$

$23010^{-7} \mathrm{~N}$ (calculated from Equation 1 and mean volume of the microspheres) while the H50

231 microspheres with a mass of $1.29 \mathrm{ng}$ experiences a buoyant force of $2.52 \times 10^{-8} \mathrm{~N}$ such that there

232 is only 1.6-fold difference in mass, but 4-fold difference in the buoyant force. Thus, the larger

233 and less dense hollow microspheres would be expected to have faster separation times in fluids

234 with similar density/viscosity properties, as seen in the kinetic data above comparing separation

235 times between the larger H20 (2.7 min in 0\% glycerol) and smaller H50 microspheres (4.7 min in

$2360 \%$ glycerol). In addition, for similarly sized microspheres in high viscosity solutions, an

237 increase in the drag force would be expected to reduce the net force and delay separation time, as

238 seen when the H20 separation time was plotted against viscosity (Fig 3). Thus, for application

239 development, this relationship was critical for selection of an appropriately sized microsphere 
240 population in order to achieve a desired separation time in a given biological matrix of known

241 viscosity.

\section{3.1.2 Size fractionation of H2O microspheres}

243 Because the buoyant force was largely size-dependent in fluids with similar

244 density/viscosity properties, we size fractionated the bulk H20 microspheres in order to obtain a

245 more homogeneous population which would enable more precise control over the buoyancy

246 properties and ultimately, the separation parameters in viscous fluids. Using wire mesh test

247 sieves with nominal mesh sizes of $105 \mu \mathrm{m}, 74 \mu \mathrm{m}, 53 \mu \mathrm{m}$, and $25 \mu \mathrm{m}$, respectively, we obtained

248 four relatively homogeneous bead populations with mean diameters ranked from smallest to

249 largest of $21 \pm 5 \mu \mathrm{m}, 38 \pm 7 \mu \mathrm{m}, 62 \pm 10 \mu \mathrm{m}$, and $81 \pm 12 \mu \mathrm{m}$ (Fig 5A, panels i - iv). Histogram

250 plots of each population are shown in Fig S1. An insufficient number of microspheres were

251 larger than the $105 \mu \mathrm{m}$ sieve size for measurement of the mean diameter in that population. The

252 vast majority of the microspheres were found in the $<25 \mu \mathrm{m}$ dia. fraction and a few of these

253 smaller particles could still be seen in the larger sieved populations due to imperfect sifting or

254 attachment to larger microspheres.

We again monitored the rate of microsphere separation for each of these size-fractionated

256 populations in PBS and 30\% glycerol as previously done for the bulk microspheres. The

257 viscosity of glycerol at $30 \% \mathrm{v} / \mathrm{v}(2.5 \mathrm{cP})$ is roughly equivalent to the viscosity or normal whole

258 blood $(3.2 \mathrm{cP})$ and plasma $(2.0 \mathrm{cP})$ [19]; therefore, it was used as a surrogate for the viscosity

259 expected in a biological fluid. As expected, separation time was inversely proportional to

260 microsphere size with the largest particles exhibiting the fastest separation times. In PBS buffer,

261 the three largest bead populations all exhibited separation times under $30 \mathrm{sec}$, while the

262 separation time for the smallest $(<25 \mu \mathrm{m}$ dia.) population was just under $2.5 \mathrm{~min}$ (Fig 5B and 
263 Table 1). In $30 \%$ glycerol, these times increased to $\leq 1 \mathrm{~min}$ for the two largest populations and

$2642.2 \mathrm{~min}$ for the $25-53 \mu \mathrm{m}$ dia. population, while the smallest set at $<25 \mu \mathrm{m}$ did not achieve

265 measurable separation over the 10-min measurement period (Fig 5C). Again, these fractionated

266 populations exhibited the same size and viscosity relationships to separation time as the bulk

267 populations, but with sharper, more exaggerated changes in OD that returned to near baseline

268 levels over shorter periods of time due to the narrower distribution of sizes present in each

269 fractionated population. Balancing the need for rapid separation versus the importance of

270 efficient interaction with pathogens in biological solutions, we selected the $\sim 38 \mu \mathrm{m}$ dia.

271 population obtained from the $25-53 \mu \mathrm{m}$ sieve range for additional functionalization and

272 pathogen binding studies.

\section{$273 \quad 3.2 \quad$ Functionalization with protein $\mathbf{G}$ and immunoglobulins}

274 Our functionalization strategy relied upon a $\mathrm{pH}$-dependent epoxy ring-opening reaction

275 that covalently linked the epoxy silane treated microspheres to biomolecular functional groups (-

$276 \mathrm{NH}_{2},-\mathrm{SH}$, and $\left.-\mathrm{OH}\right)$ in protein $\mathrm{G}($ Fig 6A) [9]. Protein $\mathrm{G}$ is an immunoglobulin (IgG)-binding

277 protein expressed in group C and G Streptococcal bacteria [20]. Since protein G binds only to

278 the Fc region of an $\mathrm{IgG}$, it was used as an orientation-specific linker to ensure that the antigen

279 binding domains of the target-specific antibody were freely available to bind pathogen targets.

280 Optimal conditions for protein $\mathrm{G}$ attachment were evaluated using a secondary GAM/Alexa-

281 Fluor@488 antibody and quantitative image analysis under various buffer conditions. A $0.1 \mathrm{M}$

282 carbonate/bicarbonate buffer at $\mathrm{pH} 9.0$ provided the most intense and uniform coating of protein

283 G on the microspheres (Supplemental Fig S2); although, conjugation was apparent for all buffer

284 and $\mathrm{pH}$ conditions tested, presumably from attachment at multiple diverse functional groups. A

285 protein $\mathrm{G}$ dilution series at steady GAM/Alexa-Fluor ${ }^{\circledR} 488$ antibody concentration exhibited a 
progressive increase in fluorescence intensity with increasing amounts of protein $\mathrm{G}$, up to 0.5

$287 \mathrm{mg} / \mathrm{ml}$ (Fig 6B). Quantitative image analysis supported this dose-dependent increase, which

288 approached $95 \%$ maximum saturation at $0.31 \mathrm{mg} / \mathrm{ml}$ of protein $\mathrm{G}$ when fit to an exponential 289 curve (Fig 6C) $\left(R^{2}=0.94\right)$. While this was an indirect method for measurement of protein $\mathrm{G}$

290 attachment, the low fluorescence intensity found in the negative control (no protein G) suggested

291 that the fluorescent antibody was not interacting with the silica surface nonspecifically, but

292 binding specifically to protein G, as was confirmed in later functional studies of antigen capture

293 without the protein G linker. We did not choose a more direct method of quantitation using

294 fluorescently labeled protein $\mathrm{G}$ because fluorophore conjugation would have utilized some or

295 even all of the same functional groups (i.e. $-\mathrm{NH}_{2}$ ) needed for reaction with the epoxy silane

296 treated microspheres.

\section{$297 \quad 3.3 \quad$ Capture and isolation of $C$. parvum oocysts in buffer and stool}

298 Using Cryptosporidium as a model pathogen/analyte, we found between $88-97 \%$ of the

299 Cryptosporidium oocysts were successfully captured and separated using the molecular buoys

300 across a wide range of clinically relevant oocyst concentrations, from $10^{3}-10^{6}$ oocysts/ml (Fig

301 7A) in buffer and stool. For example, in the presence of $10^{5}$ oocysts per $100 \mu$ sample, the

302 functionalized microspheres captured $95.1 \pm 1.9 \%$ oocysts in PBS buffer and $90.4 \pm 1.6 \%$

303 oocysts in stool versus the control microspheres without protein G, which captured less than $2 \%$

304 and $15 \%$ of the oocysts in PBS and stool, respectively. These differences between

305 positive/negative samples containing $10^{5}$ oocysts were statistically significant with $p$ values of

3060.001 for PBS and 0.007 for stool. Among the negative controls, there appeared to be a slight

307 increase in non-specific background binding in stool versus buffer possibly due to higher protein

308 content and interaction with reactive L-cysteine thiols; however, these differences were not 
statistically significant $(p=0.26)$. Furthermore, there were no significant differences in percent

310 oocysts captured by the functionalized microspheres in stool versus PBS at any of the

311 concentrations tested except in the highest $10^{5}$ samples, where the number of oocysts captured in

312 stool was slightly lower than buffer $(p=0.03)$. It is important to note that the stool matrix using

313 in this study was "watery" and meant to estimate the w/v content $(\leq 1 \mathrm{mg} / \mathrm{ml})$ that would be

314 indicative of diarrheal illness symptoms, as such, non-specific background levels may be

315 underestimated compared to clinical stool samples with a highly variable solid material content.

316 Further optimization of surface functionalization and blocking conditions may be needed for use

317 with patient-derived stool samples. Other limitations of this capture efficiency study include the

318 use of an indirect method to calculate the number of oocysts bound to the microspheres by

319 subtracting the number of un-bound oocysts counted after isolation/separation from the total

320 number input into the spiked sample (oocysts bound $_{\text {o }}=$ oocysts $_{\text {total }}-$ oocysts $\left._{\text {unbound }}\right)$. With patient-

321 derived stool samples containing an unknown number of oocysts, target release from the

322 microspheres will be critical for downstream detection and/or quantitation using flow cytometry,

323 microscopy or other technique. Methods for release may include raising or lowering the $\mathrm{pH}$ or

324 adjusting the ionic strength to disrupt the antibody-antigen interaction. However, incomplete

325 target release from the microspheres and subsequent oocyst staining/washing steps could result

326 in oocyst loss and underestimate of the number of oocysts that were bound to the microspheres,

327 which is why we chose the indirect method to measure capture efficiency in the current study.

328 We used confocal imaging to further confirm successful Cryptosporidium oocyst capture

329 and separation in pure buffer solutions. Interestingly, many of the captured oocysts were found

330 sandwiched between two of the hollow silica microspheres forming a multi-bead complex or

331 pathogen-linked network, as demonstrated in Fig 7B. Here, two fluorescent oocysts, spherical in 
332 shape and having a diameter of 4-6 $\mu \mathrm{m}$ each, were clearly seen bound to one or more

333 microsphere surfaces. Additional epi-fluorescence imaging using pre-labeled Cryptosporidium

334 oocysts, confirmed pathogen capture with target-specific antibodies bound to molecular buoys

335 coated with protein $\mathrm{G}$, but not to the blank control microspheres lacking protein $\mathrm{G}$, where

336 oocysts were only seen free-floating in solution (Fig S3).

These capture efficiency and microscopy results also suggest that the multiple protein-

338 protein interactions between protein G/antibody/target were all maintained throughout the

339 separation process and were not disrupted by frictional drag or shear forces created as the

340 particles moved through either the buffer or stool solution. A recent publication by Hsu et al.

341 described the linear relationship between the diameter of a hollow microbubble and the

342 maximum shear stress exerted upon the target-bound particle during buoyancy-assisted cell

343 separation [8]. They found that despite an increase in force with larger particles, the magnitude

344 of this force was well below $1 / 100^{\text {th }}$ of the interaction force between the microbubble-target

345 complex [8]. Thus, even with the relatively large microspheres ( $38 \mu \mathrm{m}$ in diameter) used in our

346 study, compared to the size of the target pathogen (4-6 $\mu \mathrm{m}$ in diameter) we should be well below

347 the rupture force that would cause unwanted protein-protein or target dissociation. Even when

348 we used the largest 76-105 $\mu \mathrm{m}$ fractionated microsphere population, we found similar capture

349 efficiency for Cryptosporidium oocysts ( $\leq 90 \%)$ in PBS buffer (data not shown).

350 4. CONCLUSIONS

351 In this study we have demonstrated kinetic characterization, functionalization, and

352 application of hollow silica microspheres (i.e. molecular buoys) that perform buoyancy-assisted

353 cell separation for capture and isolation of an infectious pathogen from a complex biological 
matrix. Separations were completed in less than $5 \mathrm{~min}$ with high capture efficiencies, around

$35590 \%$ or more. It is expected that this floatation-based process for rapid bioseparation will be

356 applicable to diverse protein analytes, biomarkers, and pathogens for downstream disease

357 detection and bioanalysis. Multi-plex potential exists with the use of multiple target-specific

358 antibodies functionalized on the microsphere surface, or with the use of different sized

359 microspheres each containing a unique target-specific antibody. Future work with

360 Cryptosporidium will be focused upon optimizing target-microsphere incubation times,

361 comparison against traditional methods such as magnetic affinity isolation, and establishing

362 efficacy using clinical stool samples from diarrhea patients. In addition, methods for target

363 release and downstream integration with low-cost rapid diagnostic platforms, such as paper-

364 based microfluidic devices, are currently underway.

\section{Acknowledgements}

366 Funding for this work was provided by National Science Foundation (NSF) Partnership

367 for Research and Education in Materials (DMR-1205670), the NSF's Research Triangle MRSEC

368 (DMR-1121107) and Texas State Student Undergraduate Research Fellowship.

\section{References}

370 [1] M.B. Dainiak, A. Kumar, I.Y. Galaev, B. Mattiasson, Methods in cell separations, Advances 371 in biochemical engineering/biotechnology, 106 (2007) 1-18.

372 [2] D. Pappas, K. Wang, Cellular separations: A review of new challenges in analytical 373 chemistry, Anal Chim Acta, 601 (2007) 26-35.

374 [3] M.K. Brakke, Density Gradient Centrifugation: A New Separation Technique1, Journal of the 375 American Chemical Society, 73 (1951) 1847-1848.

376 [4] J. He, M. Huang, D. Wang, Z. Zhang, G. Li, Magnetic separation techniques in sample 377 preparation for biological analysis: A review, J Pharm Biomed Anal, (2014). 
[5] O. Olsvik, T. Popovic, E. Skjerve, K.S. Cudjoe, E. Hornes, J. Ugelstad, M. Uhlen, Magnetic separation techniques in diagnostic microbiology, Clinical microbiology reviews, 7 (1994) 4338054.

381 [6] Y.R. Liou, Y.H. Wang, C.Y. Lee, P.C. Li, Buoyancy-activated cell sorting using targeted 382 biotinylated albumin microbubbles, PLoS One, 10 (2015) e0125036.

383 [7] J. Song, M. Song, T. Kang, D. Kim, L.P. Lee, Label-free density difference amplification384 based cell sorting, Biomicrofluidics, 8 (2014) 064108.

385 [8] C. Hsu, C. Chen, D. Irimia, M. Toner, Fast sorting of CD4+ T cells from whole blood using 386 glass microbubbles, Technology, 2 (2014) 1-7.

387 [9] G.T. Hermanson, Bioconjugate techniques, Academic Press, San Diego, 1996.

388

389

390

[10] J.L. Perry, J.S. Matthews, G.R. Miller, Parasite detection efficiencies of five stool concentration systems, J Clin Microbiol, 28 (1990) 1094-1097.

[11] M.W. Dryden, P. Payne, R.K. Ridley, V.E. Smith, Gastrointestinal Parasites: The Practice Guide to Accurate Diagnosis and Treatment, Suppl Compend Contin Educ Vet, 28 (2006).

[12] A.C. White, Cryptosporidiosis (Cryptosporidium hominis, Cryptosporidium parvum and Other species), in: Mandell, Bennett, Dolin (Eds.) Principles and Practice of Infectious Diseases, Churchill Livingstone, 2010, pp. 3547-3560.

[13] C.A. Schneider, W.S. Rasband, K.W. Eliceiri, NIH Image to ImageJ: 25 years of image analysis, Nat Methods, 9 (2012) 671-675.

[14] R.M.C. Dawson, Data for biochemical research, 3rd ed., Clarendon Press, Oxford, 1986.

[15] C. Rose, A. Parker, B. Jefferson, E. Cartmell, The Characterization of Feces and Urine: A Review of the Literature to Inform Advanced Treatment Technology, Critical Reviews in Environmental Science and Technology, 45 (2015) 1827-1879.

[16] J.B. Segur, H.E. Oberstar, Viscosity of Glycerol and Its Aqueous Solutions, Industrial \& Engineering Chemistry, 43 (1951) 2117-2120.

[17] L.W. Bosart, A. Snoddy, Specific gravity of glycerol, Ind Eng Chem, 20 (1928) 1377-1379.

[18] G.K. Batchelor, An Introduction to Fluid Dynamics, Cambridge University Press, Cambridge, U.K., 1999.

[19] W.F. Boron, E.L. Boulpaep, Medical Physiology, Elsevier Health Sciences, 2016.

[20] K. Kato, L.-Y. Lian, I.L. Barsukov, J.P. Derrick, H. Kim, R. Tanaka, A. Yoshino, M. Shiraishi, I. Shimada, Y. Arata, G.C.K. Roberts, Model for the complex between protein G and an antibody Fc fragment in solution, Structure, 3 (1995) 79-85. 
410

411 
412 Fig 1. Schematic of generalized "molecular buoy" concept for pathogen isolation thorough buoyancy-assisted cell 413 separation. Photograph of floated microspheres in buffer visually demonstrates separation of the hollow silica 414 microspheres from the bulk solution.

415 Fig 2. Histogram plots depicting the size distribution of (A) H20 and (B) H50 microsphere diameters measured 416 from phase-contrast microscopy images (inset).

417 Fig 3. (A) Photographs of H20 hollow silica microsphere floatation and separation at 30 sec intervals following 418 mixing. (B) Kinetic traces from optical density measurements over 10 min for H20 microspheres in buffer solutions 419 of increasing glycerol content (0-50\%) were used to determine separation times in aqueous solutions with controlled viscosity. A reference line drawn at $0.9 \mathrm{x}$ max OD change in PBS buffer, $0 \%$ glycerol, illustrates the experimentally derived separation time in PBS. Separation times closely followed the exponential relationship between glycerol content and fluid viscosity (inset, red dashed curve); (C) Optical density curves of the H50 hollow-glass microspheres in buffer and glycerol. Baseline was buffer only, without microspheres.

424 Fig 4. Force diagram and equations describing the three forces acting upon the hollow microspheres in a suspended 425 solution.

426 Fig 5. (A) Using wire mesh test sieves, four size-fractionated microsphere populations were obtained with mean diameters of (i) $21 \pm 5 \mu \mathrm{m}$, (ii) $38 \pm 7 \mu \mathrm{m}$, (iii) $62 \pm 10 \mu \mathrm{m}$, and (iv) $81 \pm 12 \mu \mathrm{m}$; (B) OD traces of each sizefractionated population in PBS and (C) 30\% glycerol demonstrate rapid separation in roughly 2 min or less for all populations, except the $<20 \mu \mathrm{m}$ dia. set, in aqueous solutions with viscosities comparable to biological fluids.

Fig 6. (A) Schematic of fully functionalized microspheres with protein $G$ followed by a target-specific IgG antibody; (B) SEM micrographs of microspheres before functionalization (panel i) and after functionalization with protein $\mathrm{G}$ (panel ii); (C) Epi-fluorescent and bright-field images of fully functionalized microspheres at increasing concentrations of protein G (panels i-vi, $0 \mathrm{mg} / \mathrm{ml}$ control, $0.01 \mathrm{mg} / \mathrm{ml}, 0.05 \mathrm{mg} / \mathrm{ml}, 0.1 \mathrm{mg} / \mathrm{ml}, 0.5 \mathrm{mg} / \mathrm{ml}$ and 1.0 $\mathrm{mg} / \mathrm{ml}$, respectively). Scale bar is $100 \mu \mathrm{m}$. Fluorescent images were brightness/contrast enhance for printing purposes only; (D) Quantitative mean fluorescence intensity (MFI) data fit to an exponential curve $\left(R^{2}=0.94\right)$.

Fig 7. (A) Capture efficiency of the molecular buoys in buffer and stool samples spiked with Cryptosporidim oocysts. Significant differences using a one-tailed t-test are shown in brackets $(p<0.05)$; (b) Confocal z-slice image of two Cryptosporidium oocysts (white arrow) sandwiched between two hollow silica microspheres in a transmitted light overlay (top) and green fluorescence channel only (bottom). 


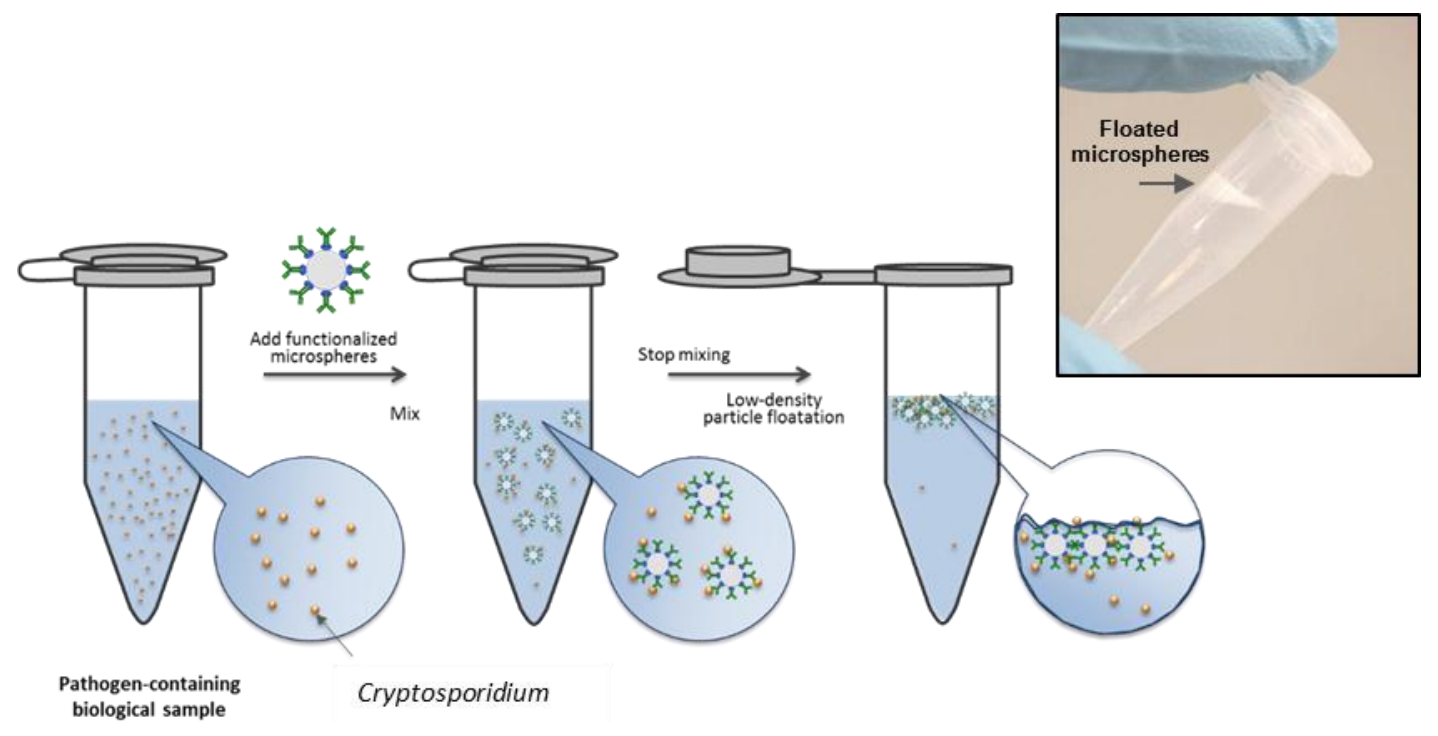

Cryptosporidium 


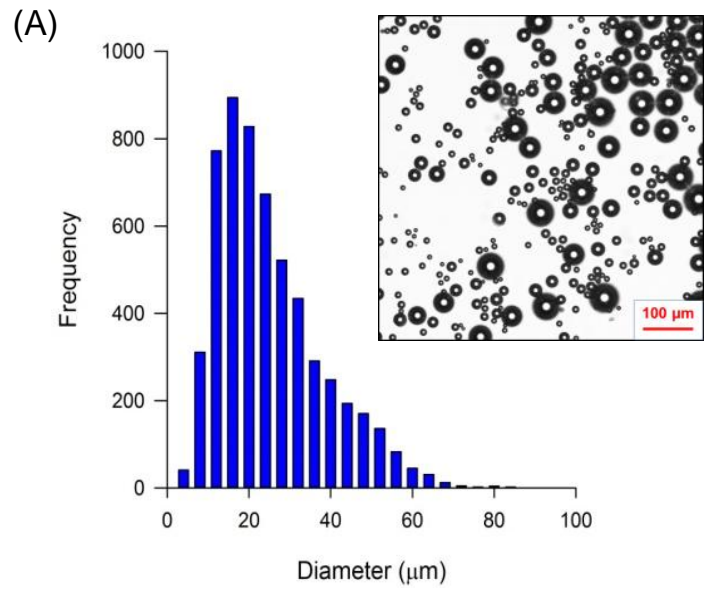

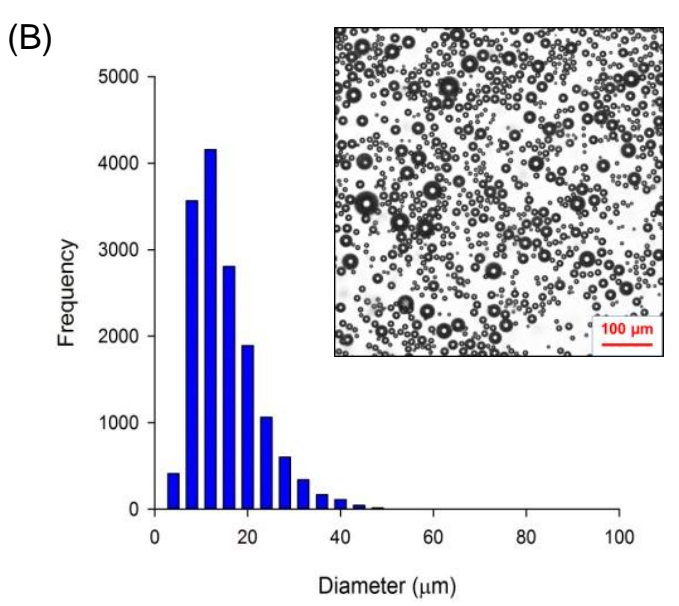


(A)
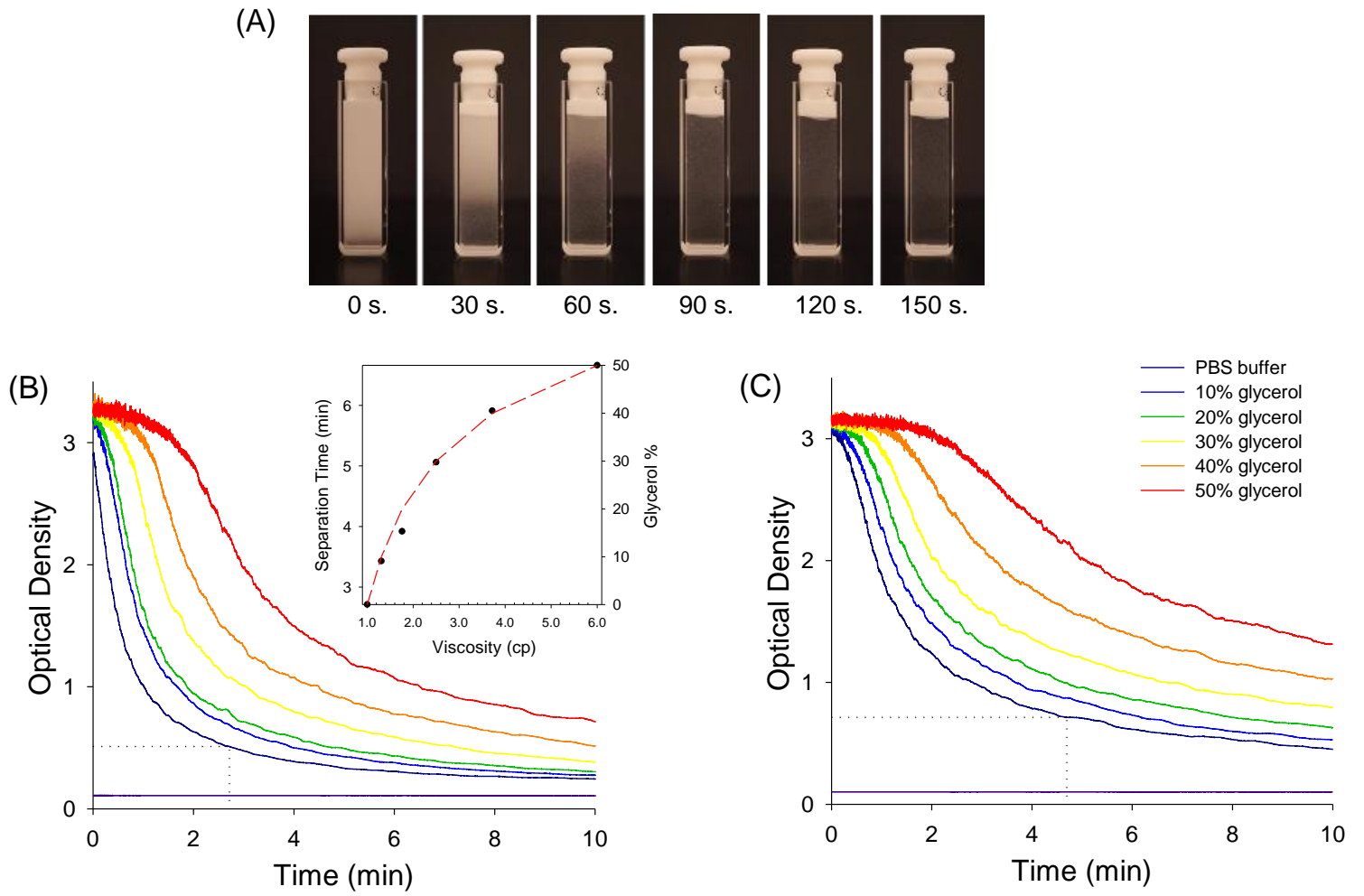


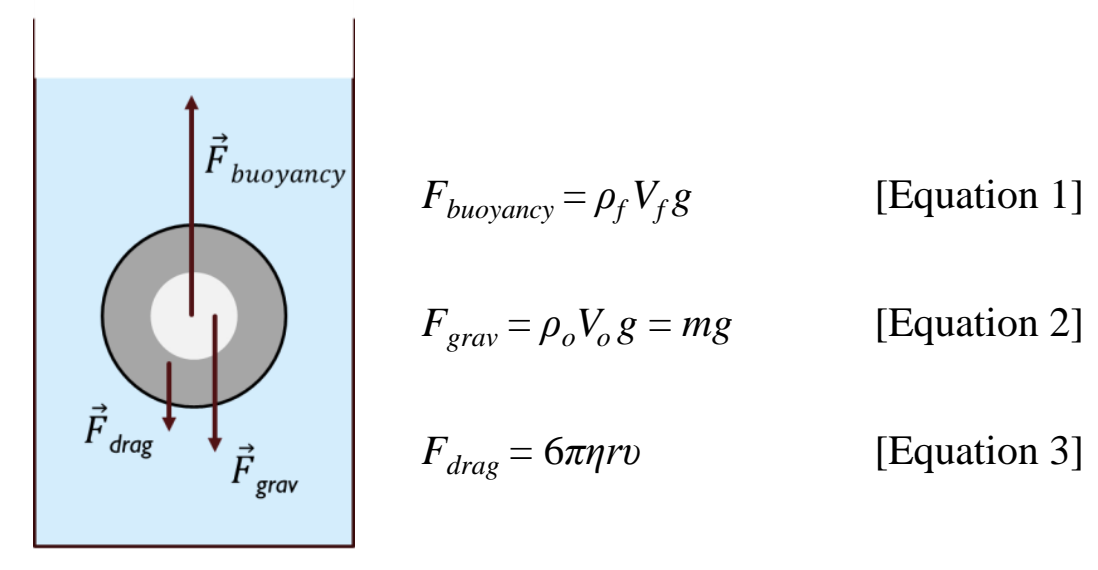


(A)

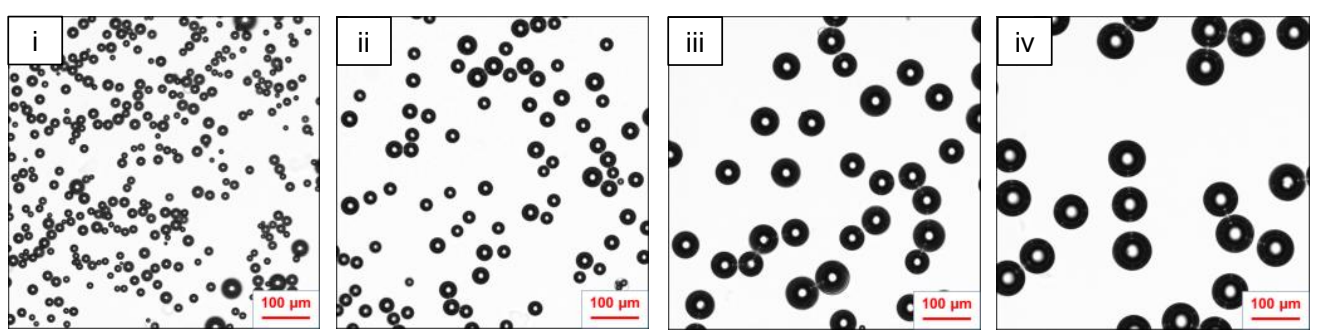

(B)

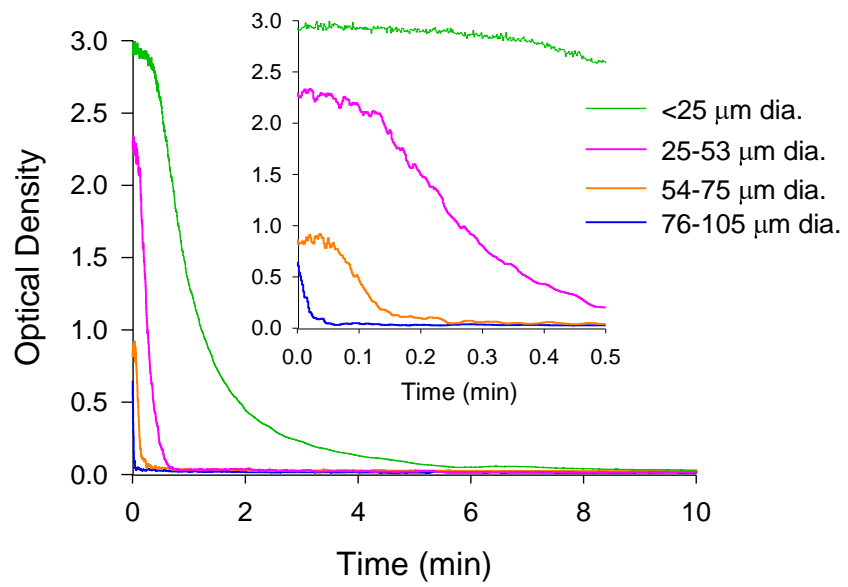

(C)

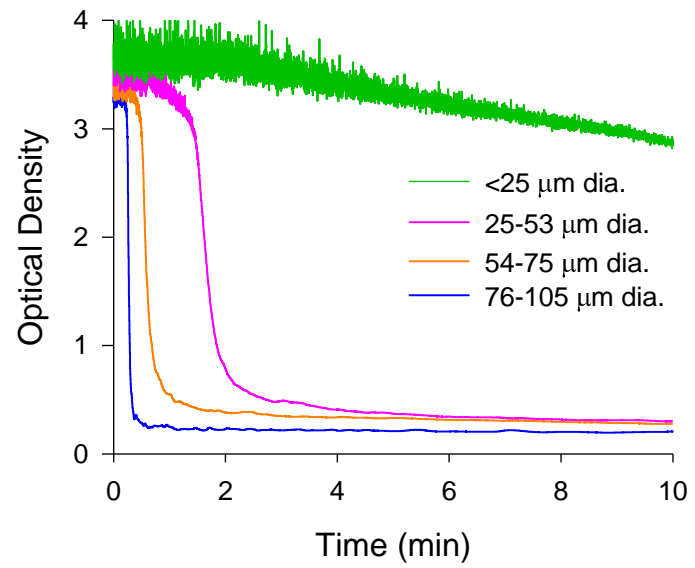


(A)

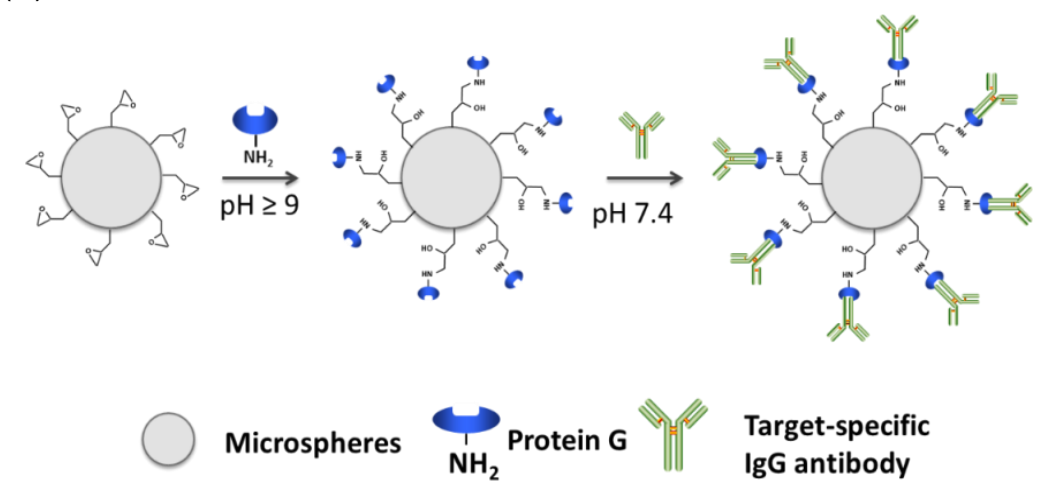

(C)

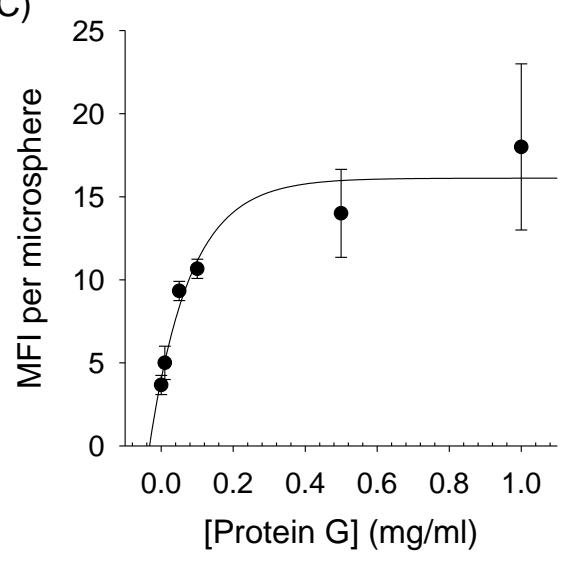

(B)

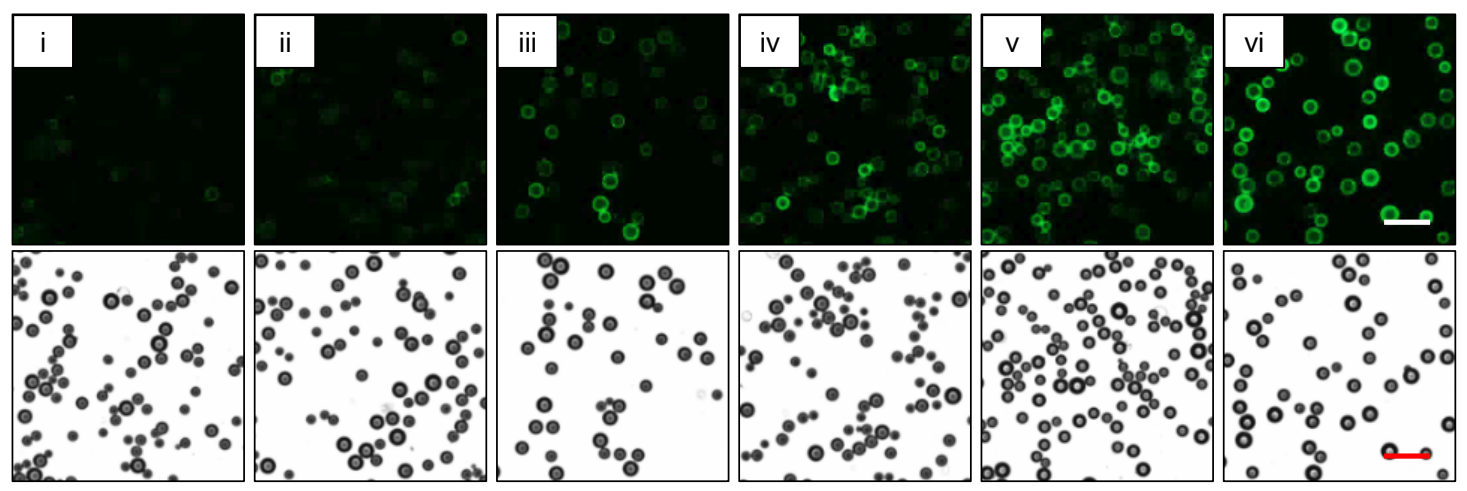


(A)

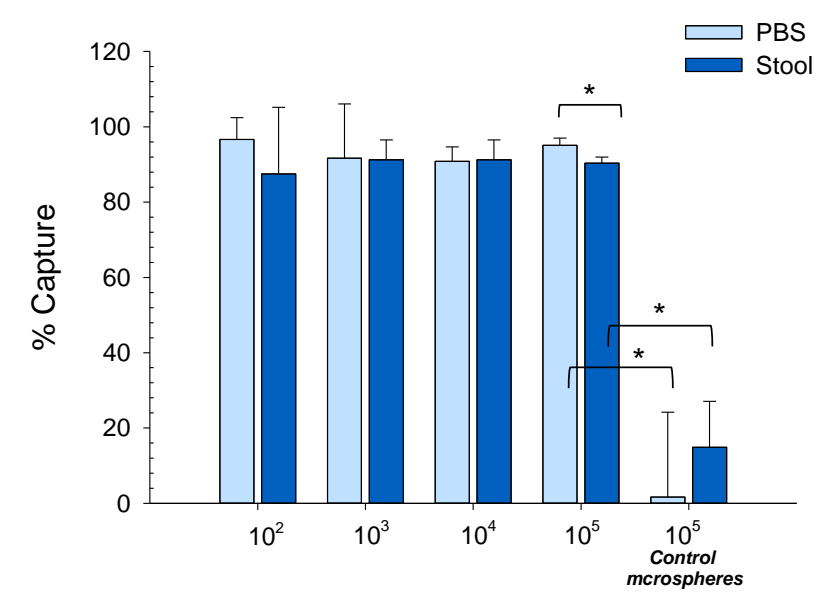

Cryptosporidim oocysts in spiked samples
(B)

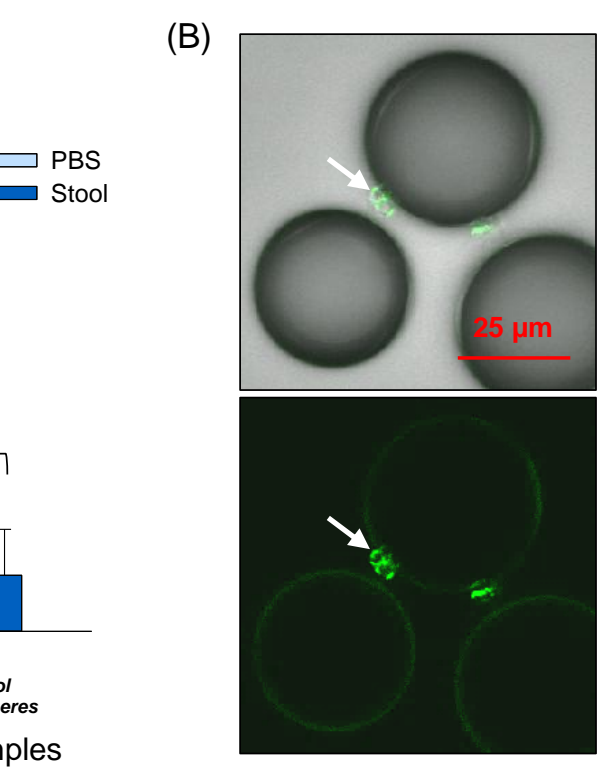

\section{Figure}

